\begin{tabular}{|c|c|}
\hline Title & $\begin{array}{l}\text { Metal-coated semiconductor nanostructures and simulation of photon extraction and coupling to optical fibers for a } \\
\text { solid-state single photon source }\end{array}$ \\
\hline Author(s) & $\begin{array}{l}\text { Suemune, Ikuo; Nakajima, Hideaki; Liu, X iangming; Odashima, Satoru; A sano, Tomoya; lijima, Hitoshi; Huh, Jae } \\
\text { Hoon; Idutsu, Y asuhiro; Sasakura, Hirotaka; Kumano, Hidekazu }\end{array}$ \\
\hline Citation & $\begin{array}{l}\text { Nanotechnology, 24(45), (455205)1-(455205)9 } \\
\text { https:/doi.org/10.1088/0957-4484/24/45/455205 }\end{array}$ \\
\hline Issue Date & 2013-11-15 \\
\hline Doc URL & http:/hdl.handle.net/2115/57451 \\
\hline Rights & (c) 2013 IOP Publishing Ltd \\
\hline Type & article (author version) \\
\hline File Information & Nanotechnology_Suemune.pdf \\
\hline
\end{tabular}

Instructions for use 


\title{
Metal-coated semiconductor nanostructures and simulation of photon extraction and coupling to optical fibers for a solid-state single-photon source
}

\author{
Ikuo Suemune, Hideaki Nakajima1, Xiangming Liu', Satoru Odashima1, \\ Tomoya Asano1, Hitoshi Iijima ${ }^{1}$, Jae-Hoon Huh ${ }^{1,2}$, Yasuhiro Idutsu', \\ Hirotaka Sasakura ${ }^{1}$, and Hidekazu Kumano ${ }^{1}$ \\ ${ }^{1}$ Research Institute for Electronic Science, Hokkaido University, Sapporo 001-0020, \\ Japan
}

E-mail: isuemune@es.hokudai.ac.jp

\begin{abstract}
We realized metal-coated semiconductor nanostructures for a stable and efficient singlephoton source (SPS) and demonstrated improved single-photon extraction efficiencies by the selection of metals and nanostructures. We demonstrate that inclination of a pillar sidewall, which changes the structure to a nanocone, is effective to improve the photon extraction efficiency with finite-difference time domain (FDTD) simulations. We demonstrate how such nanocone structures with inclined sidewalls are fabricated with reactive ion etching. With the optimized design, the photon extraction efficiency to outer airside as high as $\sim 97 \%$ generated from a quantum dot in a nanocone structure is simulated, which is the important step to realize SPS on-demand operations. We also examined the direct contact of such a metal-embedded nanocone structure to a single-mode fiber facet as a simple and practical method to prepare fiber-coupled SPS and demonstrated practical coupling efficiencies of $\sim 16 \%$ with the FDTD simulation.
\end{abstract}

\section{Introduction}

Secure and safe communication is one of the key technologies to support our present society. Quantum information and communication is guaranteed of its safety with quantum mechanical principle and is expected to be the way to realize secure information networks in near future. Quantum key distribution $(\mathrm{QKD})$ is the practical way to realize secure communication, and faint laser sources (FLS) attenuated to the single-photon level have been employed for QKD experiments. Laser sources are known to have the coherent nature and this benefits optical-fiber communications. However phase fluctuations and photon number fluctuations are related with each other under the uncertainty principle [1], and coherence generally leads to the enhancement of photon number fluctuations. Therefore photon numbers generated from FLS are under the Poisson statistics [2]. For example for an average photon number of one for each pulsed generation from FLS, the ratio of the two-photon generation rate to the onephoton generation rate is as high as $50 \%$. When the average photon number per pulse is reduced to 0.1 or 0.01 , the ratio is reduced to $5 \%$ or $0.5 \%$, respectively, and this is the reason why most of the QKD experiments have employed the reduced average photon numbers of 0.1 or less per pulse with FLSs. However, this reduces the key generation rate and also the key transmission distance [3].

Single-photon sources (SPS) have been actively studied to replace FLS mainly with semiconductor quantum dots (QDs). The drawback of semiconductors is that they generally have large refractive indices on the order of 3.5 and photons generated from QDs experience total internal reflection (TIR) at air/semiconductor interfaces. With the refractive index of 3.5, the critical angle of TIR is $\theta_{\mathrm{CR}}=16.6^{\circ}$ and the photon extraction efficiency (PEE) to airside given by $\left(1-\cos \theta_{\mathrm{CR}}\right) / 2$ is 0.021 . Therefore even if the internal quantum efficiency to emit photons from QDs is high, the photon collection efficiency (PCE) to the measurement optics remains low. To realize real SPS, the PEE to external airside and the PCE to couple photons to a guided mode in a single-mode fiber (SMF) should be as high as possible. Toward this direction, there have been active trials [4-18]. The most standard way of these trials is the microcavities based on distributed Bragg reflectors (DBRs). Pelton et al. prepared a pillar microcavity based on a pair of AlAs/GaAs DBRs and observed the PCE of $22 \%$ captured by the first lens after the photon emission from a

${ }^{2}$ present address, School of Mechanical and Advanced Materials Engineering, Ulsan National Institute of Science and Technology (UNIST), Ulsan 689-798, South Korea 
QD [4]. Strauf et al. demonstrated a high photon emission rate of $4 \mathrm{MHz}$ with an AlGaAs/GaAs DBR-based microcavity with lateral optical confinement and the high PCE of $38 \%$ was observed [6]. Gazzano et al. recently investigated essentially the same structure as the one of Ref. [4] and reported the high PCE of 79\% [18]. It is also noted that other structures such as a horn structure [5], photonic nanowire structures [7,8,16], a trumpet structure [9], coupling to a nanoantenna [10], coupling to photonic crystal waveguides [11,14], coupling to a nanophotonic directional coupler [12], coupling to a planar dielectric antenna [13], coupling to confined Tamm Plasmon modes [15], and coupling to a reversed pyramidal structure [17] have been proposed for improving the PCE.

Although the present relevant researches are mostly focused to GaAs-based SPSs emitting in the wavelength range of $880 \sim 960 \mathrm{~nm}[4,6-9,11,12,14-18]$, the extension to the longer-wavelength telecommunication band is important. There have been studies on SPS in telecommunication band with InAs QDs grown on GaAs substrates $[19,20]$. However from the viewpoint of materials, InAs QDs grown on InP substrates have the advantage due to the smaller lattice mismatch of 3.2\% rather than that of 7.2\% for InAs QDs on GaAs, where the excess strain is crucial to satisfy both conditions of high optical quality and the extension to the telecommunication band. The problem with the InP-based materials system is that efficient DBR structures are missing due to the smaller refractive-index differences among the possible combinations of layer structures. Therefore the efficient DBR-based SPS described above is difficult to extend to the telecommunication band.

One of the general methods to cope with this problem is the employment of metals that show high reflectance at semiconductor/metal interfaces in wide optical-wavelength range. Metal-coated nanocavities have been actively studied mainly for laser applications and demonstrated to operate as nanolasers with low threshold [21-28]. However studies on metal-coated nanostructures from the viewpoint of SPS applications have hardly been investigated. Maksymov et al. analyzed a metal-coated nanocylinder cavity and simulated the high PEE of $\sim 80 \%$ with the cylinder height less than $100 \mathrm{~nm}$ [29], but such an extremely shallow structure is difficult to prepare and no experimental reports have been made. We have proposed a metal-coated semiconductor nanostructure for SPS applications with improved PEE [30]. We experimentally verified single-photon emission from GaAs-based pillar structures embedded in metals [31, 32] and from GaAs-based nanocone structures [33]. In this paper, we discuss the future prospect of this kind of metal-coated nanostructures toward realizing practical on-demand SPS. The paper is arranged as follows: In section 2, we introduce our first trial to prepare the metal-coated pillar nanostructures with a cleavage method with the main emphasis on the fabrication details. From the viewpoint of the cleavage, hard metals are more convenient but they show inferior optical properties. We discuss the improvement of PEE by replacing the embedding metal to silver $(\mathrm{Ag})$. In section 3, we demonstrate that the inclination of a pillar sidewall, which changes the structure to a nanocone, is really effective to improve the PEE with systematic finite-difference time-domain (FDTD) simulations. In section 4, we demonstrate how such nanocone structures with inclined sidewalls are realized by changing the reactive ion etching (RIE) conditions from section 2 . We also demonstrate that the photon extraction efficiency to outer airside can reach as high as $\sim 97 \%$ of the photons generated from a quantum dot in a nanocone structure. In section 5, we discuss the possibility to extend the nanocone structures to the telecommunication-band InP-based semiconductor system. We also discuss the previous trials to couple SPS to SMF and examine the direct contact of such a metal-embedded nanocone structure to a SMF facet as a simple and practical method to prepare fiber-coupled SPS and demonstrate practical coupling efficiencies of $\sim 16 \%$ with the FDTD simulation. Finally we summarize and discuss the prospect of realizing practical on-demand SPS.

\section{Preparation of metal-coated semiconductor nanopillars}

We fabricated metal-coated GaAs-based nanopillars employing cleavage to remove GaAs substrates [30, 31]. InAs QDs were employed as the photon emitter and were grown with metal-organic (MO) molecular-beam epitaxy with MO sources of triethylgallium (TEGa), triethylindium (TEIn), and trisdimethylaminoarsenic (TDMAAs) for supplying Ga, In and As, respectively, to the growing semiconductor surface. An epi-ready (001) semi-insulating GaAs substrate is thermally cleaned at $560^{\circ} \mathrm{C}$ with simultaneous TDMAAs injection of $1.0 \times 10^{-4} \mathrm{~Pa}$ beam equivalent pressure [34]. After growth of a 100 -nm-thick GaAs buffer layer at $510^{\circ} \mathrm{C}$, the substrate temperature is reduced to $480^{\circ} \mathrm{C}$ for the growth of InAs QDs. Formation of InAs QDs is identified by in-situ change of reflection high-energy electron diffraction (RHEED) patterns from streaky to spotty ones. After the RHEED pattern change, the InAs growth is stopped and the temperature is reduced to $450^{\circ} \mathrm{C}$ for the growth of a 50 -nm-thick GaAs capping layer. Then the substrate temperature was reduced to room temperature. The average height, diameter, and density of InAs QDs are $6.2 \mathrm{~nm}, 32 \mathrm{~nm}$, and $4 \times 10^{9} \mathrm{~cm}^{-2}$, respectively. The grown QD sample is etched into a pillar structure with the following process. Firstly, about 300-nm-thick silicon dioxide $\left(\mathrm{SiO}_{2}\right)$ layer is deposited on the top GaAs layer surface with plasma-enhanced chemical-vapor deposition (PECVD) to increase adhesion of negative-type electron beam (EB) resist, hydrogen silsequioxane (HSQ) (Dow Corning Toray Co., Ltd.). After prebaking at $150^{\circ} \mathrm{C}$, $\mathrm{HSQ}$ resist mask is prepared with EB lithography. The resist mask pattern is transferred to the $\mathrm{SiO}_{2}$ layer with RIE employing $\mathrm{CHF}_{3}$ gas and then transferred to GaAs pillars with inductively coupled plasma (ICP)-RIE by using $\mathrm{Cl}_{2}$ and argon (Ar) mixture gas. The typical etching condition is as follows: process pressure of $0.04 \mathrm{~Pa}, \mathrm{Cl}_{2}$ and Ar flow rates of 0.1 and 0.1 standard cc per minute $(\mathrm{sccm})$, respectively, RF ICP and bias powers of $60 \mathrm{~W}$ and $100 \mathrm{~W}$, and the 

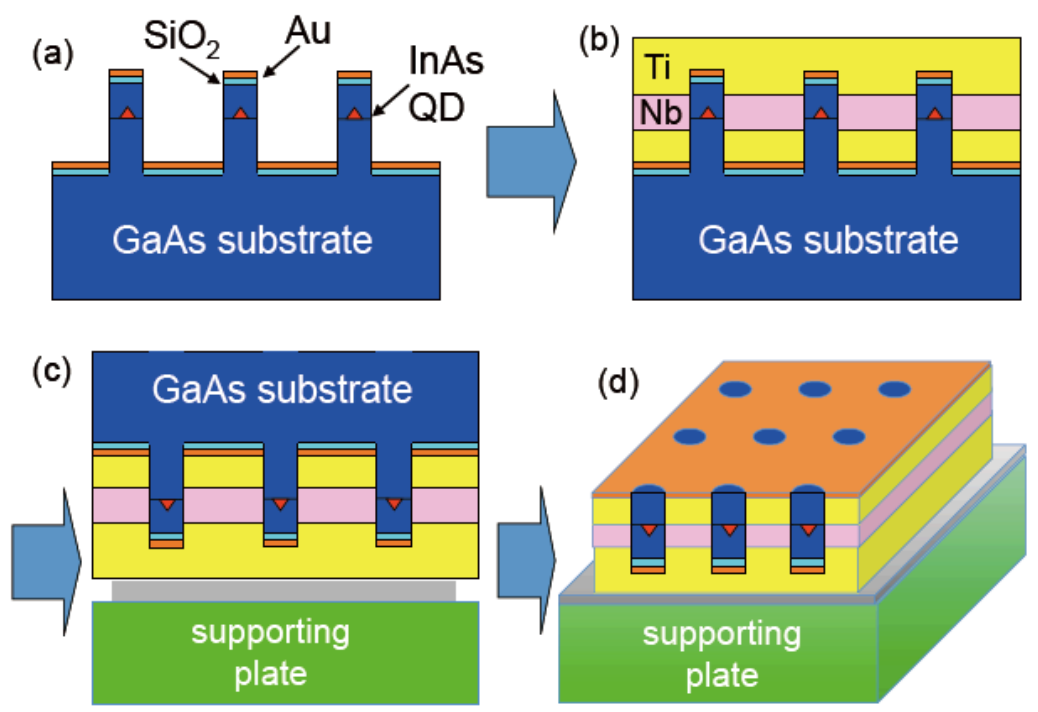

Figure 1. Schematic of the fabrication process. (a) GaAs nanopillars with InAs QDs inside are fabricated with ICPRIE and 20-nm-thick $\mathrm{SiO}_{2}$ and then a 20-nm-thick Au layers are evaporated. (b) Pillars are embedded with $\mathrm{Ti} / \mathrm{Nb}$ metal layers. (c) The sample is turned upside down and is bonded to a supporting Si plate with In $(\sim 5 \mu \mathrm{m}$ thick). (d) GaAs substrate is separated at the $\mathrm{SiO}_{2} / \mathrm{Au}$ interface and is removed from the pillars with cleavage at the bottom of the pillars.

substrate temperature of $50^{\circ} \mathrm{C}$. This results in the etching rate of $\sim 23 \mathrm{~nm} / \mathrm{min}$. The $\mathrm{SiO}_{2}$ layer remaining on top of GaAs pillars is etched off with buffered HF.

The completed pillars are typically $250 \sim 500 \mathrm{~nm}$ high and the diameter ranged from $100 \mathrm{~nm}$ to $1 \mu \mathrm{m}$. They are sequentially covered with a 20 -nm-thick $\mathrm{SiO}_{2}$ layer and then a 20 -nm-thick gold (Au) layer with vacuum evaporation as shown in figure 1(a). This combination originates from general experience that adhesion of the $\mathrm{SiO}_{2}$ and $\mathrm{Au}$ surfaces is generally weak and is expected to assist the separation of the GaAs substrate and the cleavage of nanopillars from the GaAs substrate. This is followed by the evaporation of metal layers on the surface as shown in figure 1(b). From the viewpoint of higher optical reflectivity at the metal-semiconductor interface, Au or Ag is known to exhibit high reflectivity in the $\sim 1-\mu \mathrm{m}$ emission band of InAs QDs. However in this fabrication process, mechanical hardness of the metal layers is required for the cleavage. Titanium (Ti) and niobium ( $\mathrm{Nb}$ ) have the high Vickers hardness of 970 and $1320 \mathrm{MPa}$, respectively, while $\mathrm{Au}$ and $\mathrm{Ag}$ have the much lower hardness of 216 and $251 \mathrm{MPa}$ [35]. Metal layers of $\sim 150$-nm-thick Ti/ 100-nm-thick Nb/ 150-nm-thick Ti are evaporated as shown in figure 1(b). The sample is then turned upside down and is bonded with a supporting plate (Si substrate) employing $\sim 5$ - $\mu$ m-thick Indium (In) adhesion layer (figure 1(c)). Finally, the GaAs substrate is removed from the pillars by sliding the substrate parallel to the supporting plate. The GaAs pillars are cleaved near their bottom by the lateral force during this process. The lower adhesion of the $\mathrm{SiO}_{2}$ and $\mathrm{Au}$ surfaces as well as the mechanical hardness of the $\mathrm{Ti} / \mathrm{Nb} / \mathrm{Ti}$ layers contributes to this cleavage process.

(a)

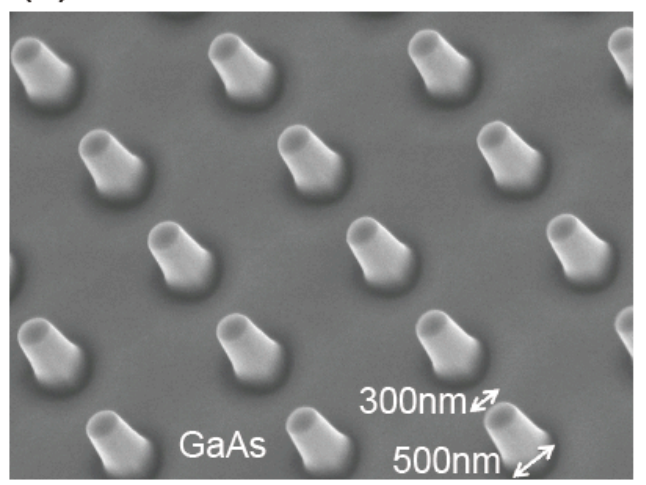

(b)

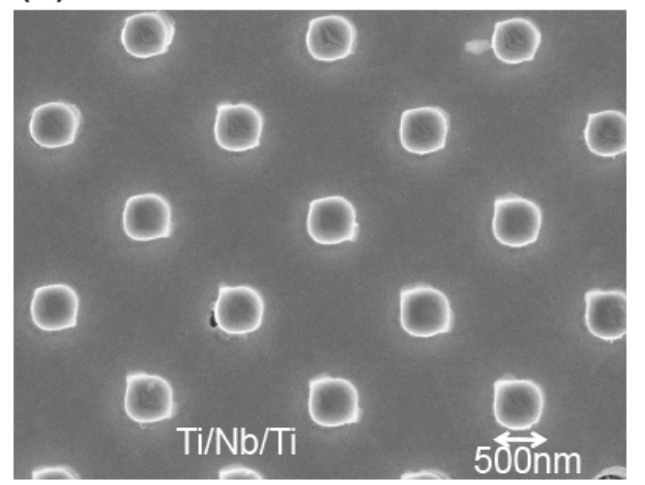

Figure 2. (a) SEM observation of fabricated GaAs-based nanopillar array. (b) SEM observation of cleaved GaAsbased nanopillar arrays embedded in $\mathrm{Ti} / \mathrm{Nb} / \mathrm{Ti}$ metal layers. 


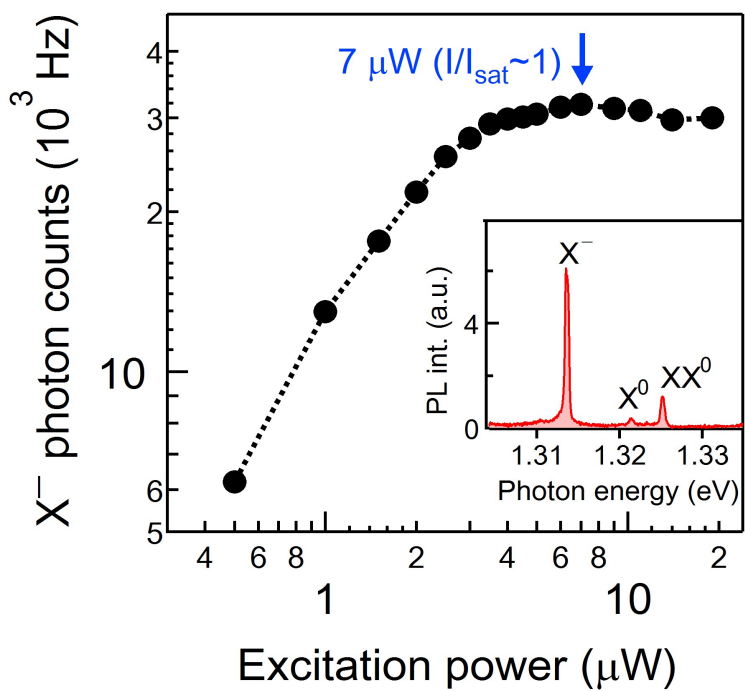

Figure 3. Luminescence spectrum measured from a single InAs QD in the GaAs pillar embedded in $\mathrm{Ti} / \mathrm{Nb} / \mathrm{Ti}$ (inset). $X, X^{0}$ and $X^{00}$ are negatively charged exciton, neutral exction, and neutral biexciton, respectively. The excitation power dependence of the $X^{-}$emission photon count rate is plotted.

Figure 2(a) is the scanning electron microscope (SEM) image of a two-dimensional (2-D) array of GaAs pillars prepared with ICP-RIE. The pillars in this example are arranged with the periodicity of $1.5 \mu \mathrm{m}$. Each pillar is $\sim 500$ $\mathrm{nm}$ high and is slightly tapered. The diameter is $\sim 300 \mathrm{~nm}$ at the top and $\sim 500 \mathrm{~nm}$ at the bottom. Figure 2(b) is the 2$\mathrm{D}$ array of GaAs pillars embedded in the $\mathrm{Ti} / \mathrm{Nb} / \mathrm{Ti}$ metal layers. The diameter of each pillar surface is $\sim 500 \mathrm{~nm}$ and corresponds to the diameter at the pillar bottom in figure 2(a). Although some pillars show surface features, the flat pillar surfaces demonstrate the successful cleavage near the pillar bottom to prepare the metal-embedded pillar structures.

Optical properties were studied on one of the nanopillars with the pillar height of $250 \mathrm{~nm}$ and the diameter of 500 $\mathrm{nm}$. A sharp emission line was observed at the wavelength of $944.3 \mathrm{~nm}(1.313 \mathrm{eV})$ at $20 \mathrm{~K}$ and was assigned as the negatively charged exciton $\left(X^{-}\right)$and the other two peaks as neutral exciton $\left(X^{0}\right)$ and neutral biexciton $\left(X X^{0}\right)$ [31] as shown in the inset of figure 3. The InAs QDs were located at the plane $50 \mathrm{~nm}$ above the meta/semiconductor bottom interface. Photon autocorrelation measurements were performed with the Hanbury-Brown and Twiss (HBT) setup [36]. Incident photons are divided into two optical paths with a non-polarized beam splitter and are detected with a single-photon counting module (SPCM) in each path. The SPCM quantum efficiency at the detection wavelength of

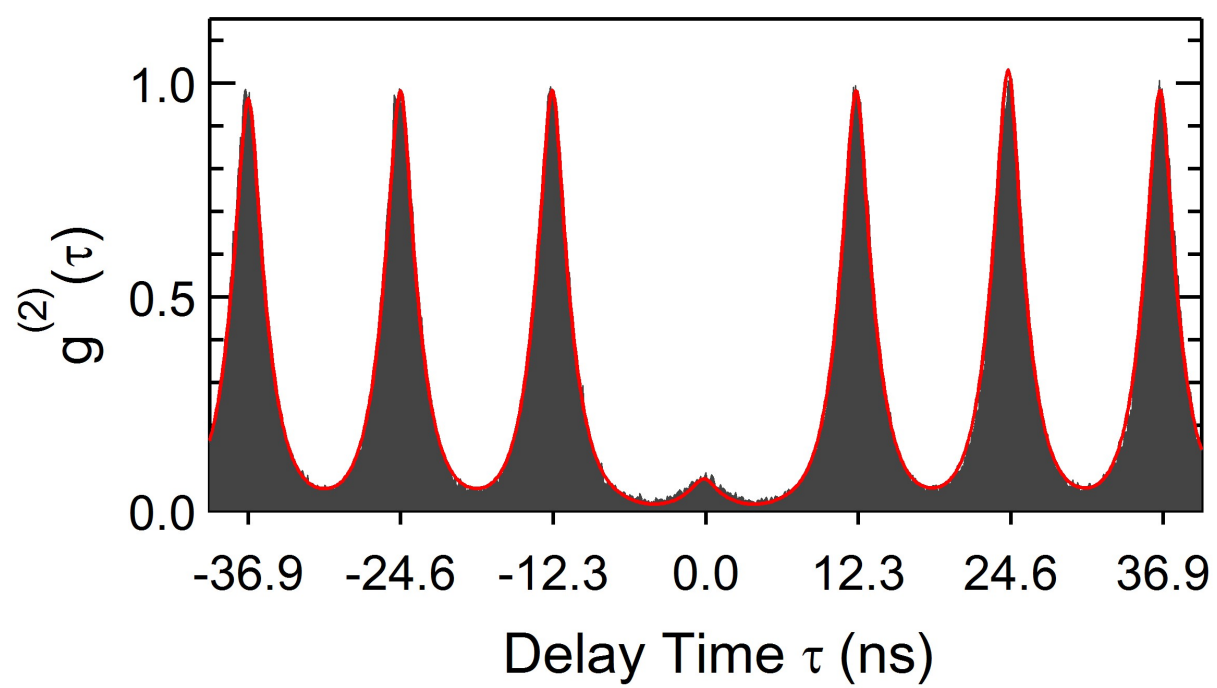

Figure 4. Second-order intensity autocorrelation function, $g^{(2)}(\tau)$, measured with the photon coincidence counts in the HBT setup. The excitation power was set at the saturation level of $7 \mu \mathrm{W}$. The solid curve in red is the theoretical fit to the measured data to get the $\mathrm{g}^{(2)}(0)$ value with the known formula given in Ref. 38 . 
$\sim 945 \mathrm{~nm}$ was $\sim 27 \%$ and the dark count rate was $\sim 100$ counts per second (cps). Photon detection in one path supplies a start signal to a multi-channel analyzer (MCA), while another photon detection in the other path supplies a stop signal to the MCA. Counting the start-stop events gives the histogram of photon coincidence counts against the relative delay time $\tau$ of the two paths, which is proportional to the second-order intensity autocorrelation function, $g^{(2)}(\tau)$ [37]. The measurements show the clear dip at the zero delay time as shown in figure 4 and the anti-bunching of the generated photons, that is, the single-photon emission, is demonstrated. The $g^{(2)}(0)$ value is related to the residual two-photon generation probability of SPS and is the important evaluation index for the application to quantum cryptography [3], and it was 0.08 in this case.

The photon detection rate measured at the saturation level of $7 \mu \mathrm{W}$ was $31,800 \mathrm{cps}$ as shown in figure 3 . Then the single photon detection rate is estimated to be $30,500 \mathrm{cps}$ by multiplying the factor of $\left[1-g^{(2)}(0)\right]^{1 / 2}$ to correct the multi-photon emission probability [4]. The photon detection efficiency of our measurement setup at this stage was 0.0045 and therefore the single-photon flux at the first photon collection lens with the numerical aperture (NA) of 0.42 is $6.8 \mathrm{MHz}$. The excitation pulse repetition rate is $82 \mathrm{MHz}$ which is the repetition frequency of a mode-locked Ti:Sapphire laser with the pulse duration of $\sim 5$ ps used in this experiment, and the PCE with the first lens is estimated to be $8.3 \%$.

The above sample structure was prepared with cleavage to remove the GaAs substrate employing the hard metals of $\mathrm{Nb}$ and $\mathrm{Ti}$. However their optical reflectivity is low at the GaAs/metal interfaces. Ag is a softer metal but shows higher optical reflectivity. We prepared longer pillars with the height of $750 \mathrm{~nm}$ so that the pillars withstand the cleavage force with Ag-embedding. We also introduced a 5-nm-thick silane coupling agent (Siliconize L-25) at the metal/ $\mathrm{SiO}_{2}$ interface. This coupling agent forms stable chemical bonds to the $\mathrm{SiO}_{2}$ surface, while the other surface is hydrogen-terminated and shows the hydrophobic nature to the Ag surface. This made it easy to separate GaAs substrates from the Ag surface and made it possible to prepare the Ag-embedded nanopillar structures with high yield [32]. We studied the photon extraction properties on an Ag-embedded GaAs-based pillar with the height of $750 \mathrm{~nm}$ and the bottom and top diameters of 400 and $620 \mathrm{~nm}$, respectively. In this case we observed the higher photon detection rate of $72,000 \mathrm{cps}$. With the photon autocorrelation measurements, the $g^{(2)}(0)$ was measured to be 0.06 at the photon emission saturation level. Therefore the single-photon detection rate is re-evaluated to be $69,800 \mathrm{cps}$. The photon detection efficiency of the measurement setup was 0.0051 in this case and the single-photon flux at the first collection lens is $13.7 \mathrm{MHz}$. With the excitation pulse repetition rate of $76 \mathrm{MHz}$ of the mode-locked laser for this measurement, the PCE by the first lens is estimated to be $18 \%$. This increase of the PCE is attributed to the higher optical reflectivity of $\mathrm{Ag}$ replacing $\mathrm{Nb} / \mathrm{Ti}$.

\section{FDTD simulation of photon extraction efficiency: role of sidewalls}

For pursuing the higher efficiency to extract photons from the emitters, we have worked on the FDTD simulations. The FDTD simulation is a computational electrodynamic modeling technique to calculate the Maxwell's differential equations directly by replacing the partial differential terms with finite difference equations and is known to give

(a)

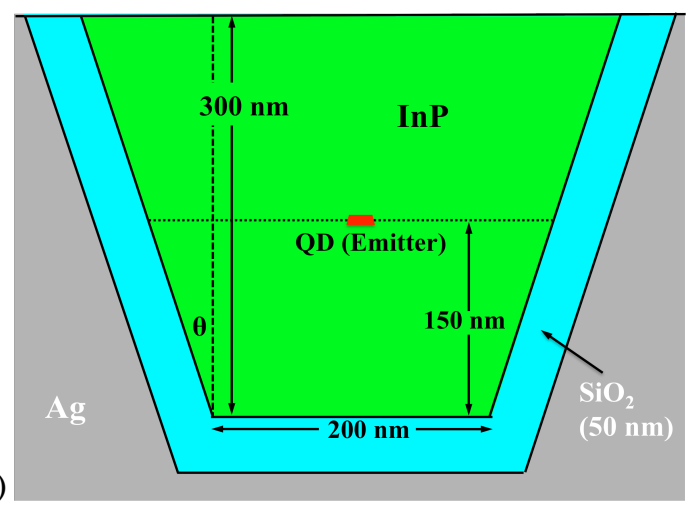

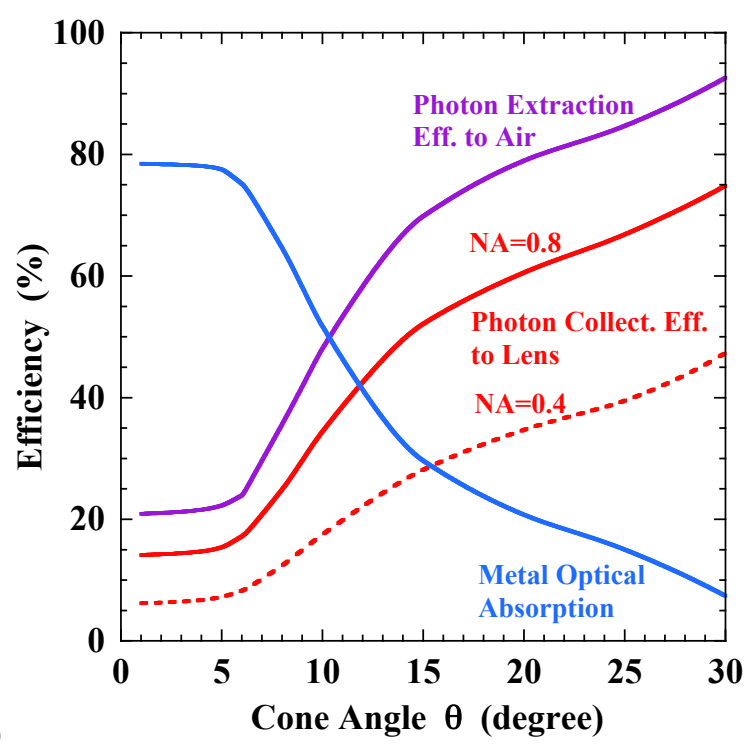

(b)

Figure 5. (a) Schematic of the nanocone structure and (b) the calculated cone angle (sidewall inclination angle) dependence of the photon extraction and absorption efficiencies. 
accurate results with a small enough grid size [39,40]. In the present simulation the grid size of $1 / 60$ of $\lambda / n_{\text {semiconductor }}$ is employed. Here the wavelength $\lambda$ is assumed at $1550 \mathrm{~nm}$ in the telecommunication band. The grid size is $8.2 \mathrm{~nm}$ for InP-based nanostructures and is small enough for high-accuracy three-dimensional modeling.

The schematic of the calculated structure is shown in figure 5(a). Among several parameters of the pillar structures, here we focus to the sidewall inclination angle dependence of the photon extraction properties. The height direction of the nanostructure is assigned as the $\mathrm{z}$ direction and the top and bottom planes of the nanostructures as the $\mathrm{x}-\mathrm{y}$ plane. The height is given as $300 \mathrm{~nm}$ and the QD emitter is set at the center of the plane separated by $150 \mathrm{~nm}$ from the bottom plane. The photon emission from the QD is modeled with a small (5-nm long) dipole directed in the y direction. A 50-nm-thick $\mathrm{SiO}_{2}$ layer is inserted at the interface between the semiconductor ( $\left.\mathrm{InP}\right)$ and embedding $\mathrm{Ag}$ to prevent the plasmon loss. This plasmon loss is especially sensitive to the Ag surface roughness [41] and care should be taken to smoothen the $\mathrm{SiO}_{2}$ surface to prevent the light absorption.

Figure 5(b) shows the calculated sidewall inclination angle $\theta$ dependence of the photon absorption and extraction efficiencies for the constant bottom plane diameter of $200 \mathrm{~nm}$. The inclination of the sidewall of a nanopillar will change the structure into a nanocone with truncated apex, and therefore we will call the structure as nanocone. In this structure the optical absorption of Ag dominates for the small cone angle $\theta$ but is reduced for the larger cone angle. With the decrease of the metal absorption, the PEE toward the outer airside increases. The PCE to a lens with the numerical aperture (NA) of 0.4 (detection angle of $\pm 23.6^{\circ}$ ) or $0.8\left( \pm 53.1^{\circ}\right)$ is also plotted. This simulation result demonstrates that higher PEE and PCE are expected with nanocones with the larger sidewall inclination angles.

\section{Silver-embedded nanocones}

Based on the above simulation on the improvement of the PEE with the larger sidewall inclination angle, we tried to fabricate the nanocone structures employing the same InAs QD/GaAs sample as the one used in Sec. 2. The sample surface is covered with the 300-nm-thick $\mathrm{SiO}_{2}$ with PECVD. The HSQ-resist circular mask was prepared with the EB lithography on the $\mathrm{SiO}_{2}$ surface and the resist pattern was transferred to the $\mathrm{SiO}_{2}$ layer with RIE. The etching condition is as follows: process pressure of $2.0 \mathrm{~Pa}, \mathrm{CHF}_{3}$ flow rate of $10 \mathrm{sccm}$, radio frequency (RF) power of $175 \mathrm{~W}$, and etching time of $7 \mathrm{~min}$. and half. This gives the etching rate of $\sim 40 \mathrm{~nm} / \mathrm{s}$. This results in a side-etched $\mathrm{SiO}_{2} \mathrm{mask}$, that is, the periphery of the top 200-nm region has the taper angle of $\sim 30^{\circ}$ and the lower 100-nm region retained a cylinder with the vertical sidewall.

Employing the nanocone-shaped $\mathrm{SiO}_{2}$ mask, we prepared GaAs-based nanocone structures with ICP-RIE by using $\mathrm{Cl}_{2}$ and argon (Ar) mixture gas. The typical etching condition is as follows: process pressure of $0.5 \mathrm{~Pa}, \mathrm{Cl}_{2}$ and $\mathrm{Ar}$ flow rates of 0.2 and $1.8 \mathrm{sccm}$, respectively, RF ICP and bias powers of $150 \mathrm{~W}$ each, and the substrate temperature of $50^{\circ} \mathrm{C}$. One of the etched nanocones is shown in figure $6(\mathrm{a})$. The etching time is $2 \mathrm{~min}$. and half and the nanocone height is $\sim 500 \mathrm{~nm}$. The $\mathrm{SiO}_{2}$ mask diameter transferred from the computer-aided design (CAD) pattern of the EB lithography is $300 \mathrm{~nm}$. During etching of 500-nm-deep GaAs, side etching occurred by $\sim 120 \mathrm{~nm}$ from the periphery of the $\mathrm{SiO}_{2}$ mask and the flat top surface with the diameter of $\sim 60 \mathrm{~nm}$ remained. The sidewall angle is $23.5^{\circ}$ on average [41]. The higher process pressure than that employed in section 2 increases the scattering of the incident ions and assists the chemical reactions on the etching surface. Ar generally acts as a buffer gas in plasma etching and can modify the etching profile [42-44] with the relative increase of the Ar ratio. The formation of the nanocone structure in the present work is also assisted with the nanocone-shaped $\mathrm{SiO}_{2}$ mask. With the CAD pattern diameters larger than $\sim 300 \mathrm{~nm}$, top flat areas increase in the prepared nanocone structures.
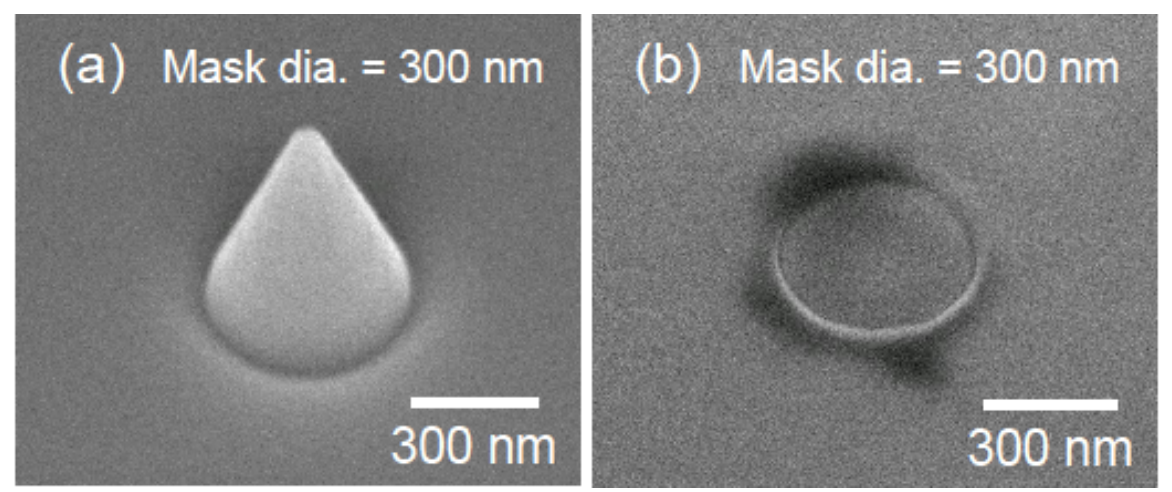

Figure 6. (a) As-etched GaAs-based nanocone prepared with ICP-RIE. The height is $\sim 500 \mathrm{~nm}$. (b) GaAs-based nanocone with the size similar to (a) after embedded in Ag. The center circle is the bottom of the GaAs nanocone and the other surface is $\mathrm{Ag}$ covered with 60 -nm-thick $\mathrm{SiO}_{2}$. 
These nanocone structures were uniformly covered with a 60-nm-thick $\mathrm{SiO}_{2}$ layer employing PECVD and then covered with 3- $\mu$ m-thick Ag layer employing an electron-beam evaporator. The Ag surface was pasted to a $\sim 0.5-$ mm-thick cover glass with a ultra-violet (UV) resin at room temperature, and the contact was hardened with UVlight irradiation. The $\sim 450-\mu \mathrm{m}$-thick GaAs substrate was thinned to $\sim 30 \mu \mathrm{m}$ with mechanical polishing. The remaining GaAs substrate was etched with ICP-RIE with an accelerated etching rate of $\sim 400 \mathrm{~nm} / \mathrm{min}$. The etching condition is as follows: process pressure of $0.2 \mathrm{~Pa}, \mathrm{Cl}_{2}$ and $\mathrm{Ar}$ flow rates of 1.0 and $0.1 \mathrm{sccm}$, respectively, $\mathrm{RF}$ ICP and bias powers of 150 and $300 \mathrm{~W}$, and the substrate temperature of $50^{\circ} \mathrm{C}$. One of the nanocones embedded in $\mathrm{Ag}$ is shown in figure 6(b). The CAD pattern diameter is the same as that of figure 6(a). The Ag surface is covered with the 60-nm-thick $\mathrm{SiO}_{2}$ and is protected from the $\mathrm{Cl}$ plasma during the ICP-RIE. The darkened area around the embedded GaAs nanocone surface was formed during the SEM observation and is not essential to the prepared samples.

The single photon emission properties were examined on one of the nanocones prepared with the CAD pattern diameter of $900 \mathrm{~nm}$. The dominant peak was the $X$ emission accompanied with the smaller $X^{0}$ peak as shown in the inset of figure 7. The photon detection rate of the $X$ emission was 196,000 cps as shown in figure 7 . With the photon autocorrelation measurement, the $g^{(2)}(0)$ was measured to be 0.30 at the saturation level excitation. Then the reevaluated single photon detection rate is $164,000 \mathrm{cps}$. The photon detection efficiency of our measurement setup in this case was 0.010 and therefore the single-photon flux at the first photon collection lens with the NA of 0.42 is 16.4 $\mathrm{MHz}$. With the excitation pulse repetition rate of $76 \mathrm{MHz}$, the PCE by the first lens is estimated to be $22 \%$. Since $X$ and $X^{0}$ emissions are anti-bunching with each other [33], the inclusion of the both emissions results in the PCE of $24.6 \%$. This further improved PEE is attributed to the difference of the nanocone with the inclined sidewall and the previous nano-pillars with nearly vertical sidewalls.

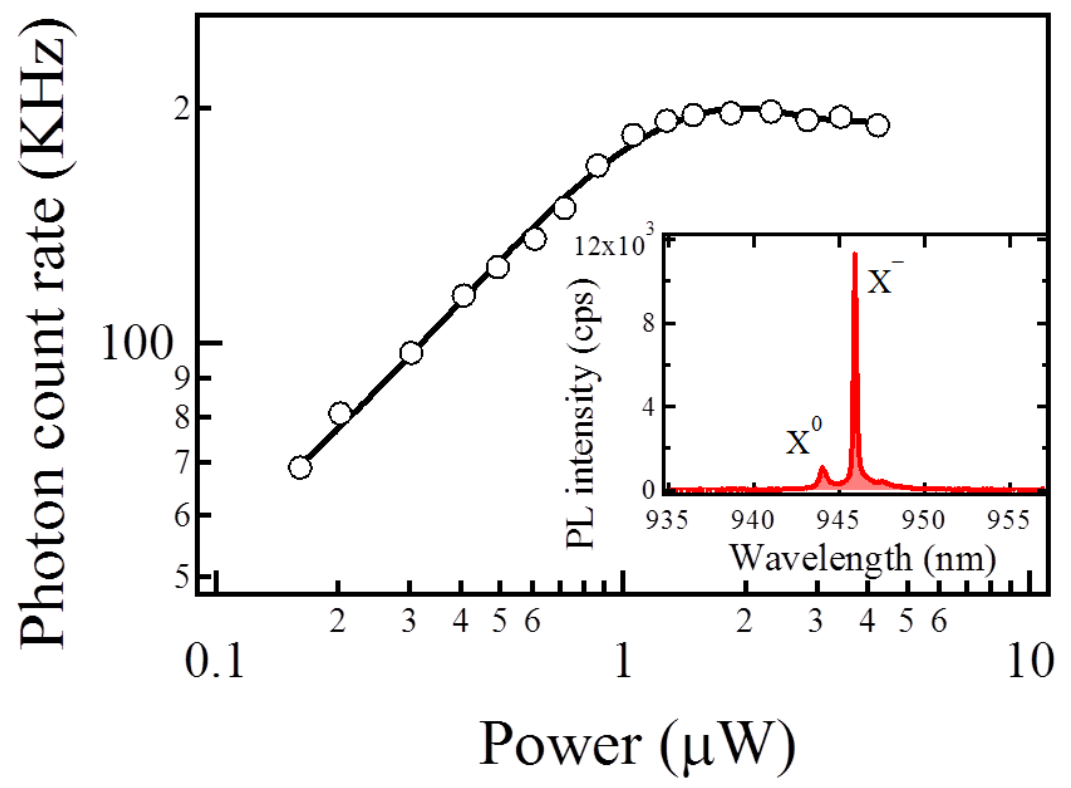

Figure 7. Luminescence spectrum measured from a single InAs QD in the GaAs nanocone embedded in Ag (inset). The excitation power dependence of the $\mathrm{X}^{-}$emission photon count rate is plotted.

Toward the further improvement of the PEE and PCE of the nanocone structures, we have worked on the FDTD simulations of the Ag-embedded GaAs nanocone. Since 3-D FDTD simulation needs extended too long simulation time for a larger-sized nanocone, we assumed the height of $300 \mathrm{~nm}$, the smaller bottom surface diameter of $100 \mathrm{~nm}$, and the sidewall angle of $24^{\circ}$. This results in the top surface diameter of $367 \mathrm{~nm}$. Since the main factor that influences the PEE is the metal optical absorption, we calculated the dependence on the interface $\mathrm{SiO}_{2}$ layer thickness dependence. Based on the fabricated nanocones, the QD emitter was assigned at the center of the plane 50 $\mathrm{nm}$ separated from the bottom surface. The emission wavelength is given at $950 \mathrm{~nm}$ in accordance with experiments. The simulation results are summarized in figure 8. One of the main findings is that the PEE to airside remains low without the insertion of the interface $\mathrm{SiO}_{2}$ layer. This is because surface plasmon is excited at the $\mathrm{Ag} / \mathrm{GaAs}$ interface and induces excess metal optical absorption (identified with the simulated electric field localized to the $\mathrm{Ag} / \mathrm{GaAs}$ interface, not shown). With the increase of the $\mathrm{SiO}_{2}$ layer thickness more than $\sim 50 \mathrm{~nm}$, the metal optical absorption is much reduced and the PEE to outer airside can approach $\sim 97 \%$. PCE to a lens with NA=0.4 is limited to below 


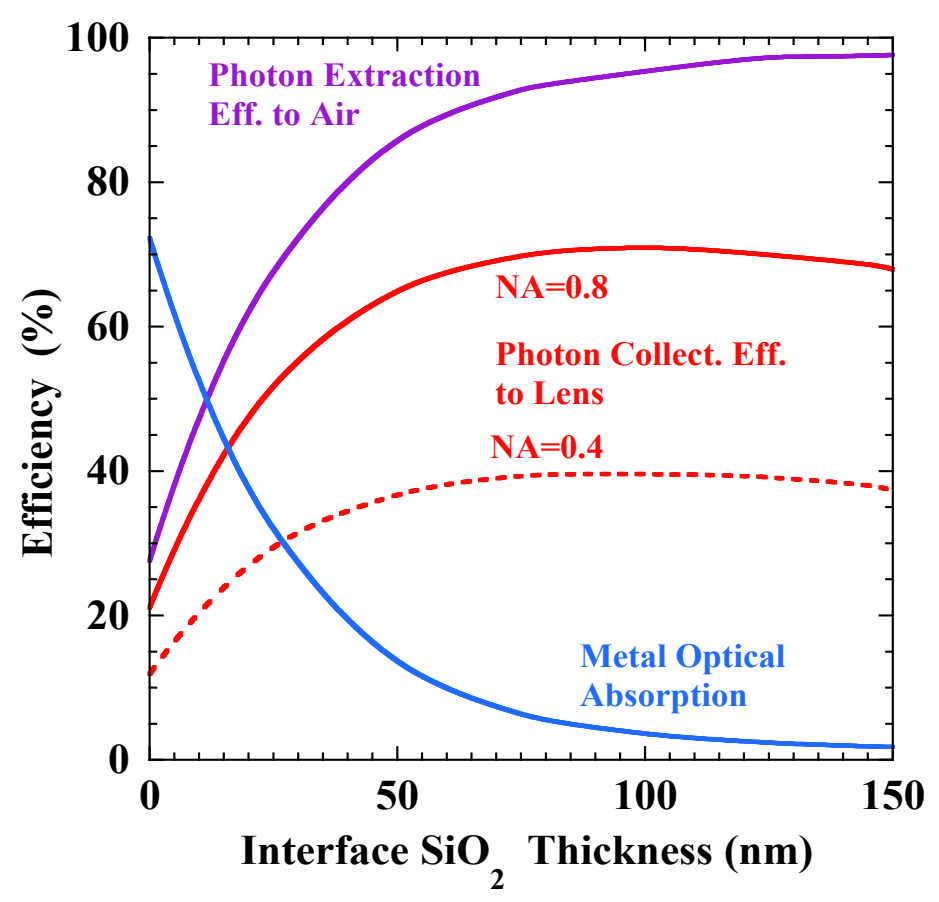

Figure 8. FDTD simulation of the interface $\mathrm{SiO}_{2}$ thickness dependence of a Ag-embedded GaAs-based nanocone. The PEE and PCE as well as the metal optical absorption are summarized.

$40 \%$. Our measured PCE of $\sim 25 \%$ is lower than this value, but this is not surprising since the QD position can not be fixed at the center of the nanostructure at present. However figure 8 shows that much higher PCE is expected by replacing the lens with $\mathrm{NA}=0.4$ to the one with $\mathrm{NA}=0.8$. For realizing the PCE close to PEE, the directionality of the emitted photon beam should be controlled by modifying the sidewall structure. One of the examples is the horn structure [5].

\section{Coupling to single-mode fiber}

For practical applications of SPS to QKD, efficient coupling of SPS to SMF is important. There have been extensive studies to couple nanoemitters to SMF. One of the efficient methods is the use of tapered nanofibers [45-47]. Tapered nanofibers have the tapered fiber diameter reduced to the order of $1 \mu \mathrm{m}$ or less to extend the optical field to the external air region, where nanoemitters are in contact on the fiber surface. This method has achieved efficient photon coupling of $22 \%$ to a SMF with the fiber diameter of $350 \mathrm{~nm}$ at the wavelength of $780 \mathrm{~nm}$ [47]. One of the remaining issues for their practical applications is the mechanical stability of the fiber system. Another interesting trial has been reported by pasting a photonic crystal (PC) nanocavity on a SMF facet [48]. In this method, the size difference of SMF cores $(5 \sim 10 \mu \mathrm{m}$ in diameter) and PC nanocavities $(\sim 1 \mu \mathrm{m}$ in size $)$ is the remaining issue. Since many QDs are present outside the nanocavity area, their photon emission couples to the core of the SMF and this tends to mask the single photon emission from a single QD in the nanocavity. As a preliminary trial, we demonstrated a QD emitter coupled to SMF facets in bidirectional configuration [49].

In addition to the efficient photon coupling to SMF, preparation of efficient telecommunication-band SPS is also necessary. The horn structure based on InAs QDs/InP is the one example [5]. In the previous section, we have demonstrated the fabrication of Ag-embedded GaAs nanocones and their efficient photon extraction properties. We are trying to apply this method to InP-based nanostructures for SMF telecommunication-band applications. InPbased nanostructures are normally processed with ICP-RIE at elevated temperature to enhance $\mathrm{InCl}_{\mathrm{x}}$ desorption from processing surfaces [42]. However we found that InP-based semiconductors show higher tendency of inclined sidewalls than GaAs with ICP-RIE at room temperature [50]. An example of the prepared InP-based nanocones is shown in figure 9. The etching condition is as follows: process pressure of $0.2 \mathrm{~Pa}, \mathrm{Cl}_{2}$ and Ar flow rates of 0.8 and $0.1 \mathrm{sccm}$, respectively, RF ICP and bias powers of 100 and $300 \mathrm{~W}$, and the sample not intentionally heated. The height of the nanocones is $\sim 350 \mathrm{~nm}$ and the most of the nanocone is $\operatorname{In}_{0.53} \mathrm{Al}_{0.22} \mathrm{Ga}_{0.25} \mathrm{As}$ barriers grown on a (311)B InP substrate. The etching rate is $\sim 230 \mathrm{~nm} / \mathrm{min}$ and the sidewall inclination angle is $\sim 20^{\circ}$. The inclination angle increases with the lower process pressure [51]. One of the etched nanostructure was embedded in Ag and bright emission at $1550 \mathrm{~nm}$ was observed. The comparison of the luminescence intensity with the as-etched sample showed 


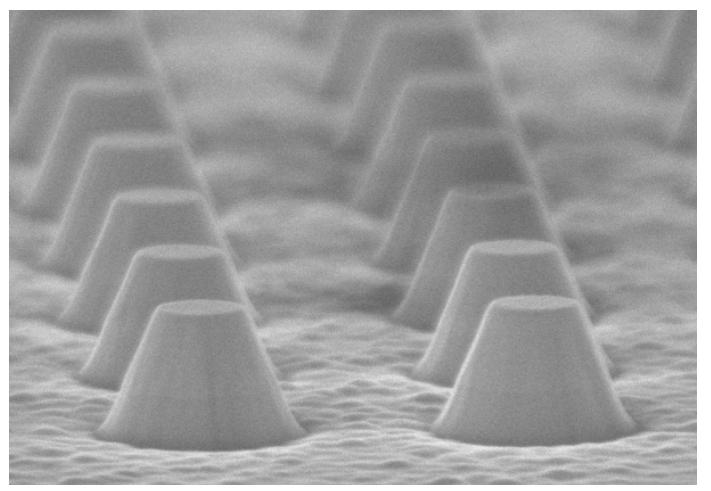

Figure 9. SEM image of etched InP-based nanocones (the substrate is InP and the nanocone is mostly $\left.\mathrm{In}_{0.53} \mathrm{Al}_{0.22} \mathrm{Ga}_{0.25} \mathrm{As}\right)$.

$\sim 30$-times enhancement under the same excitation power [51]. For the quantitative estimation of PCE, reproducible and accurate estimation of the optical coupling efficiencies to SMF connected to superconducting single-photon detectors is necessary and it is now under progress.

Based on these situations, we study the coupling efficiency of the Ag-embedded nanocone to a SMF with the FDTD simulation at the wavelength of $1550 \mathrm{~nm}$. The structure of the nanocone is essentially the same as the one dealt with in figure 5 . The nanocone bottom plane diameter of $100 \mathrm{~nm}$ and the sidewall inclination angle of $26^{\circ}$ are assumed and this results in the top plane diameter of $393 \mathrm{~nm}$ for the height of $300 \mathrm{~nm}$. A photon source with rightcircular polarization is assumed at the center of the plane $150 \mathrm{~nm}$ apart from the bottom plane. The circularly polarized photon source is constructed by the superposition of two nanoscale dipoles directed in the $\mathrm{x}$ and $\mathrm{y}$ directions with $\pi / 2$ phase shift with each other. The Ag/nanocone interface insulator was assumed as 60-nm-thick $\mathrm{Si}_{3} \mathrm{~N}_{4}$ in this case. This InP-based nanocone is pasted to the facet of a SMF with the core diameter of $4 \mu \mathrm{m}$ and the refractive-index difference of 0.042 (relative difference of $2.9 \%$ ) as shown in figure $10(\mathrm{a})$. The simulated propagation field (electric filed in the y direction) is shown in figure 10(b). For a 100-nm-thick paste resin at the interface between the fiber edge and the Ag-embedded nanocone, the PEE from the nanocone to the fiber side is $78.3 \%$ and the PCE to the guided mode is $15.9 \%$. Although the simulated electric field distribution shown in figure 10 (b) suggests efficient coupling to the guided mode, substantial part of the generated photons leaks away into the cladding area. It is noted that the increase of the paste resin to the thickness of $1 \mu \mathrm{m}$ reduces the PCE to $11.3 \%$ and therefore the efficiency is critically dependent on how the nanocone is pasted to the fiber edge. For the higher coupling efficiencies, further effort to collimate the far-field pattern of the nanocone output beam is necessary. One of the method is to modify the sidewall to a parabolic one that will collimate the output photon directionality [5].
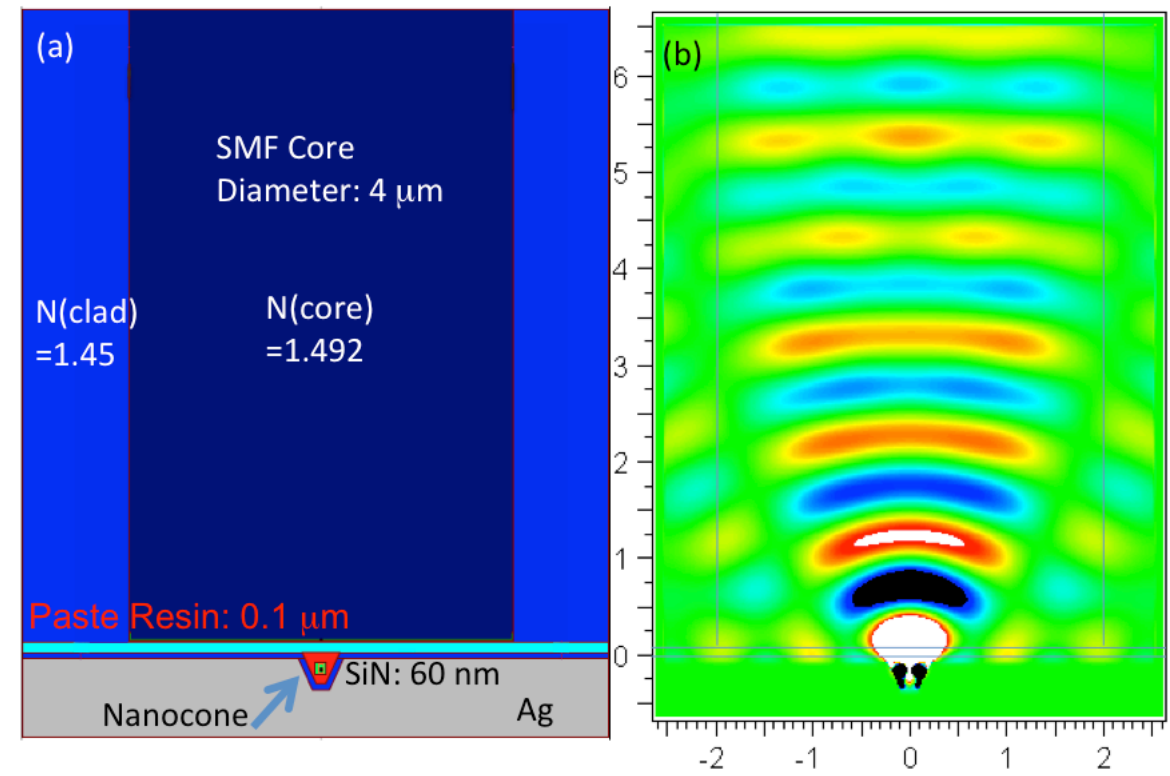

Figure 10. (a) Schematic of InAs/InP QD nanocone embedded in Ag pasted at the SMF facet. (b) Simulated light propagation along the core of the SMF. 


\section{Conclusion}

We demonstrated the successful fabrication of metal-embedded GaAs nanostructures. Photon extraction efficiency was initially $8.3 \%$ and was low because of employing hard metals due to the fabrication process. The replacement of the metal with $\mathrm{Ag}$ improved the efficiency up to $18 \%$ because of the higher optical reflectivity at the $\mathrm{Ag} / \mathrm{GaAs}$ interface. Higher efficiency of $\sim 25 \%$ was achieved with the modification of GaAs nanopillars to nanocones. All these measurements were performed with photon collection lens with $\mathrm{NA}=0.42$. Therefore the higher efficiency is expected by replacing the lens with the one with higher NA as is demonstrated in figures 5(b) and 8. FDTD simulation of the Ag-embedded nanocone showed that the photon extraction to airside can approach as high as $\sim 97 \%$. The photon collection efficiency to the first lens is limited to $\sim 70 \%$ with the presently assumed straight nanocone sidewall. The efficiency will be further improved by modifying the sidewall to a parabolic one that will collimate the output photon directionality. Direct contact of such a nanocone structure to the facet of a single-mode fiber was also simulated and the photon extraction efficiency of $\sim 78 \%$ and the coupling efficiency of $\sim 16 \%$ to the guided mode was obtained. This coupling efficiency will also be improved by incorporating the parabolic-shaped nanocone sidewalls to the Ag-embedded SPS. These results demonstrate that metal-coated semiconductor nanostructures offer practical and efficient fiber-based single photon sources that will approach to the on-demand operations with the further optimization of the semiconductor nanostructures.

\section{Acknowledgments}

The authors wish to thank Kouich Akahane, Yasuhiro Matsuo, and Kuniharu Ijiro for the relevant collaborative research. This work was partially supported by the Grand-in-Aid for Scientific Research (S), No.24226007, Nanotechnology Platform by the Ministry of Education, Culture, Sports, Science and Technology, and SCOPE (Strategic Information and Communications R\&D Promotion Programme) from the Ministry of Internal Affairs and Communications, Japan.

\section{References}

[1] Korolkova N V and Chirkin A S 1998 Quantum Electron. 28287

[2] Loudon R 1973The Quantum Theory of Light (Oxford: Clarendon)

[3] Takesue H, Nam S W, Zhang Q, Hadfield R H, Honjo T, Tamaki K and Yamamoto Y 2007 Nature Photon. 1343

[4] Pelton M, Santor C, Vuckovic J, Zhang B, Solomon G S, Plant J and Yamamoto Y 2002 Phys. Rev. Lett. 89 233602

[5] Takemoto K, Takatsu M, Hirose S, Yokoyama N, Sakuma Y, Usuki T, Miyazaki T and Arakawa Y 2007 J. Appl. Phys. 101081720

[6] Strauf S, Stoltz N G, Rakher M T, Coldren L A, Petroff P M and Bouwmeester D 2007 Nature Photon. 1704

[7] Fiedler I, Sauvan C, Hugonin J P, Lalanne P, Claudon J and Gerard J M 2009 Opt. Express 172095

[8] Claudon J, Bleuse J, Makik N S, Bazin M, Jaffrennou P, Gregersen N, Sauvan C, Lalanne P and Gerard J M 2010 Nature Photon. 4174

[9] Gregersen N, Nielsen T R, Mork J, Claudon J and Gerard J M 2010 Opt. Express 1821204

[10] Curto A G, Volpe G, Taminiau T H, Kreuzer M P, Quidant R, van Hulst N F 2010 Science 329930

[11] Schwagmann A, Kalliakos S, Farrer I, Griffiths J P, Jones G A C, Ritchie D A and Shields A J 2011 99, 261108

[12] Davanco M, Rakher M T, Wegscheider W, Schuh D, Badolato A and Srinivasan K 2011 Appl. Phys. Lett. 99 121101

[13] Lee K G, Chen X W, Eghlidi H, Kukura P, Lettow R, Renn A, Sandoghdar V and Gotzinger S Nat. Photon. 5 166

[14] Enderlin A, Ota Y, Ohta R, Kumagai N, Ishida S, Iwamoto S and Arakawa Y 2012 Phys. Rev. B 86075314

[15] Gazzano O, Vasconcellos S M, Gauthron K, Symonds C, Voisin P, Bellessa J, Lemaitre A and Senellart P 2012 Appl. Phys. Lett. 100232111

[16] Reimer M E, Bulgarini G, Akopian N, Hocevar M, Bavinck M B, Verhijen M A, Bakkers E P A M, Kouwenhoven L P and Zwiller V 2012 Nature Commun. 3737

[17] Rulke D, Schaadt D M, Kalt H and Hetterich M 2012 Appl. Phys. Lett. 100251101 
[18] Gazzano O, Vasconcellos S M, Arnold C, Nowak A, Galopin E, Sagnes I, Lanco L, Lemaitre A and Senellart P 2013 Nature Commun. 41425

[19] Ward M B, Karimov O Z, Unitt D C, Yuan Z L, See P, Gevaux D G, Shields A J, Atkinson P and Ritchie D A 2005 Appl. Phys. Lett. 86201111

[20] Intallura P M, Ward M B, Karimov O Z, Yuan Z L, See P, Shields A J, Atkinson P and Ritchie D A 2007 Appl. Phys. Lett. 91161103

[21] Hill M T, Oei Y-S, Smalbrugge B, Zhu Y, Vries T J, Veldhoven P J, Otten F W M, Eijkemans T J, Turkiewicz J P, Waardt H D, Geluk E J, Kwon S-H, Lee Y-H, Notzel R and Smit M K 2007 Nat. Photon. 1589

[22] Lu C-Y, Chang S-W, Yang S-H and Chuang S L 2009 Appl. Phys. Lett. 95233507

[23] Hill M T, Marell M, Leong E S P, Smalbrugge B, Zhu Y, Sun M, Veldhoven P J, Geluk E J, Karouta F, Oei Y-S, Notzel R, Ning C-Z and Smit M K 2009 Opt. Express 1711107

[24] Yu K, Lakhani A and Wu M C 2010 Opt. Express 188790

[25] Nezhad M P, Simic A, Bondarenko O, Slutsky B, Mizrahi A, Feng L, Lomakin V and Fainman Y 2010 Nat. Photon. 4395

[26] Lu C-Y, Chang S-W, Chuang S L, Germann T D and Bimberg D 2010 Appl. Phys. Lett. 96251101

[27] Lu C-Y, Chang S-W, Chuang S L, Germann T D, Pohl U W and Bimberg D 2011 Semicond. Sci. Technol. 26 014012

[28] Lee J H, Khajavikhan M, Simic A, Gu Q, Bondarenko O, Slutsky B, Nezhad M P and Fainman Y 2011 Opt. Express 1921524

[29] Maksymov I S, Besbes M, Hugonin J P, Yang J, Beveratos A, Sagnes I, Robert-Philip and Lalane P 2010 Phys. Rev. Lett. 105180502

[30] Kumano H, Nakajima H, Ekuni S, Idutsu Y, Sasakura H and Suemune I 2010 Advances in Mathematical Phys. 20101

[31] Nakajima H, Ekuni S, Kumano H, Idutsu Y, Miyamura S, Kato D, Ida S, Sasakura H and Suemune I 2011 Phys. Stat. Solidi C 8337

[32] Kumano H, Nakajima H, Iijima H, Odashima S, Matsuo Y, Ijiro K and Suemune I 2013 Appl. Phys. Express 6 062801

[33] Liu X, Asano T, Odashima S, Nakajima H, Sasakura H, Kumano H and Suemune I 2013 Appl. Phys. Lett. 102 131114

[34] Idutsu Y, Miyamura S and Suemune I 2009 Phys. Stat. Solid. C 6868

[35] Smith R L and Sandland G E 1922 Proc. Institut. Mechan. Eng. 1623

[36] Hanbury-Brown R and Twiss R Q 1956 Nature 17727

[37] Lounis B, Bechtel H A, Gerion D, Alivisatos P and Moerner W E 2000 Chem. Phys. Lett. 329399

[38] Nakajima H, Kumano H, Iijima H and Suemune I 2012 Appl. Phys. Lett. 101161107

[39] Yee K 1966 IEEE Trans. Antenn. Propag. 14302

[40] Taflove A 1980 IEEE Trans. Electromagn. Compat. EMC-22 191

[41] Kim S-K, Ee H-S, Choi W, Kwon S-H, Kang J-H, Kim Y-H, Kwon H and Park H-G 2011 Appl. Phys. Lett. 98 011109

[42] Yoon S F, Ng T K and Zheng H Q 2001 Thin Solid Films 394250

[43] Lee H W, Kim M, Min N-K, Efremov A, Lee C-W and Kwon K-H 2008 Jpn. J. Appl. Phys. 476917.

[44] Weibin Q and Jiaxian W 2012 J. Semiconductors 33026001

[45] Fujiwara M, Toubaru K, Noda T, Zhao H-Q and Takeuchi S 2011 Nano Lett. 114362

[46] Davanco M, Rakher M T, Wegscheider W, Schuh D and Badolato A 2011 Appl. Phys. Lett. 99121101

[47] Yalla R, Kien F L, Morinaga M and Hakuta K 2012 Phys. Rev. Lett. 109063602

[48] Shambat G, Provine J, Rivoire K, Sarmiento T, Harris J and Vuckovic J 2011 Appl. Phys. Lett. 99191102

[49] Sasakura H, Liu X, Odashima S, Kumano H, Muto S and Suemune I 2013 Appl. Phys. Express 6065203

[50] Huh J-H, Hermannstaedter C, Akahane K, Sasakura H, Jahan N A, Sasaki M and Suemune I 2011 Jpn. J. Appl. Phys. 50 06GG02

[51] Huh J-H, Hermannstaedter C, Akahane K, Jahan N A, Sasaki M and Suemune I 2012 Jpn. J. Appl. Phys. 51 06FF12 